\title{
Design Simulation of Top ITO Gratings to Improve Light Transmission for Gallium Nitride LEDs
}

\author{
Xiaomin Jin ${ }^{1,3}$, Simeon Trieu ${ }^{1}$, Fei Wang ${ }^{2}$, Bei Zhang ${ }^{3}$, Tao Dai ${ }^{3}$, Xiangning Kang ${ }^{3}$, and Guoyi \\ Zhang $^{3}$ \\ ${ }^{1}$ Electrical Engineering Department, California Polytechnic State University, \\ San Luis Obispo, CA 93407, USA, xjin@calpoly.edu \\ ${ }^{2}$ Electrical Engineering Department, California State University at Long Beach, Long Beach, \\ CA 90840, USA \\ ${ }^{3}$ State Key Laboratory for Mesoscopic Physics and Department of Physics, Peking University, \\ Beijing 100871,PR China
}

\begin{abstract}
We present simulation results of the indium tin oxide (ITO) top diffraction grating using a rigorous couple wave analysis (RCWA) for GaN LEDs. We study three different nano-structure patterns: cylindrical pillar grating, conical pillar grating, and cylindrical nano-hole grating. We show the light transmission improvement with nano-grating designs and present design-charts for the nano-hole grating.
\end{abstract}

\section{Introduction}

The conventional GaN-based light-emitting diodes (LEDs) have a low light extraction efficiency caused by the total internal reflection. A common way to solve this light trapping is to etch a periodic nano-structure at the light extraction surface and/or the bottom reflective layer of the LEDs [1]-[4].

For commercial applications, low cost and simplicity in fabrication are desired. It has been demonstrated by Peking University in 2008 that 32\% of light extraction enhancement was achieved by using the triangular patterned encapsulated Flip-chip (FC) GaN LEDs compared to C-LEDs [5]. In this design, the surface gratings and the encapsulation of a polymer can be simultaneously accomplished in a single procedure; additionally it provides low height profile. Therefore, it can realize thin and low cost LED package. However, our pervious work focuses on mirco-scale grating patterns. To obtain design guidelines and understand design parameters in the nano-scale, we developed a three-dimensional (3D) rigorous couple wave analysis (RCWA) GaN-based light-emitting diode (LED) grating model to study top diffraction grating design using Rsoft DiffMod [6]. In the paper, we also provide a design charts for the improvement of the LED light extraction and optimize the nano-hole-patterned polymer top grating design.

In summary, we present a three-dimensional (3D) rigorous couple wave analysis (RCWA) GaN-based light-emitting diode (LED) grating model to study the top indium tin oxide (ITO) diffraction grating performance at $460 \mathrm{~nm}$ wavelength in order to improve the GaN LED light transmission efficiency.

\section{Design simulation}

The core algorithm of the model is based on RCWA and enhanced with modal transmission line theory. The RCWA [7] [8] represents the electromagnetic fields as a sum of coupled waves. A periodic permittivity function is represented using Fourier harmonics. Each coupled wave is related to a Fourier harmonic, allowing the full vectorial Maxwell's equations to be solved in the Fourier domain. Currently, plane wave incidence is assumed and material is lossless to simplify the calculation.

Usually it is not very practical to fabricate all kinds of the top ITO textures or patterns to select the optimized structure [9][10]. Therefore, we simulate three typical gratings: cylindrical pillar grating, conical pillar grating, and cylindrical nanohole grating, as shown in Fig. 1(a)-(c). The detailed GaN LED layer structure is also presented in Fig. 2. In our device design, it is very important to keep a $30 \mathrm{~nm}$-fixed ITO thinckness at bottom of the grating. This fixed thickness is used to prevent P-GaN layer from being damaged in the etching process and 
protect the overall device charaterization. Furthermore, the 30nm-bottom ITO layer also acts as the current injection layer to protect the LED I-V charaterization from being effected by the nanostructure.

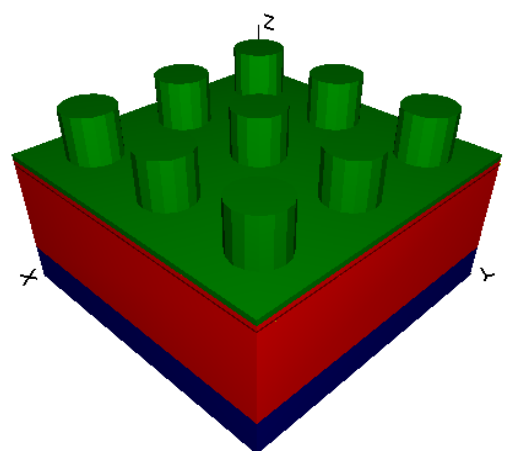

(a)

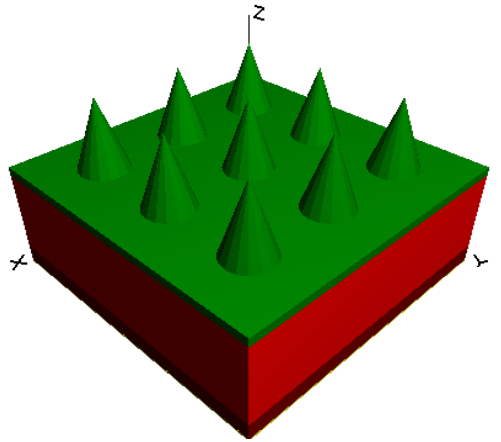

(b)

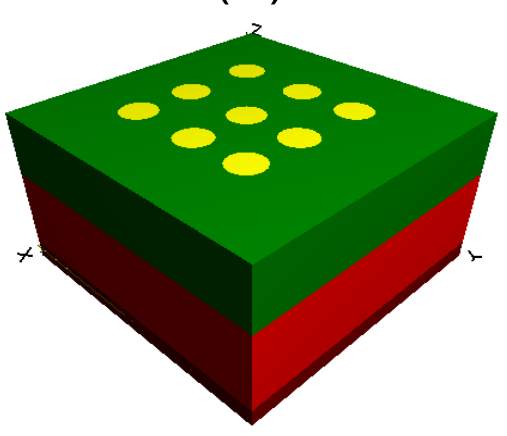

(c)

Fig. 1 The schematic diagrams of the top grating simulation a) cylindrical pillar grating, b) conical pillar grating, and c) cylindrical nano-hole grating

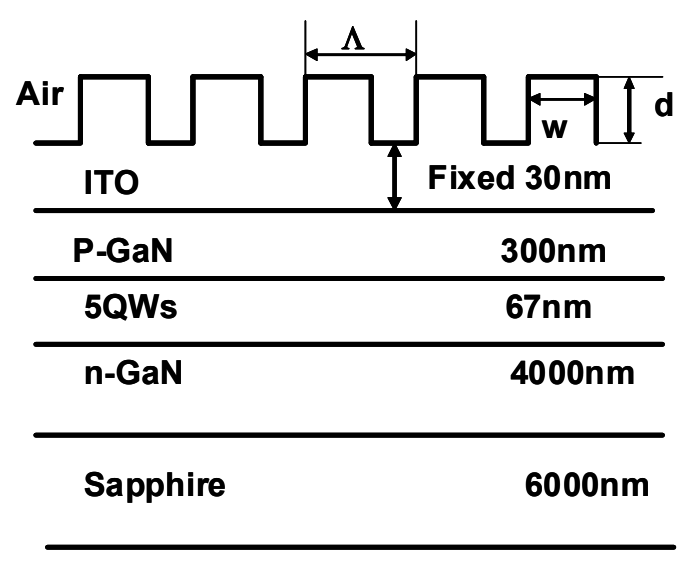

Fig. 2 The detailed LED layer structure in the models.

In the grating simulation model, there are three major parameters that affect the light extraction: grating period $(\Lambda)$, grating height $(d)$, and bottom width (w). First, according to our fabrication capability, we choose a large grating period $\Lambda=300 \mathrm{~nm}$ for the initial calculation [11]. We sweep the other two parameters $(\mathrm{d}$ and $\mathrm{w})$ and their possible permutations. The simulation results are shown in Fig. 2 (a)-(c). For the grating height (d) smaller than $60 \mathrm{~nm}$, cylindrical pillar grating has the best performance. All three cases have the best performance at larger $\mathrm{w}$ value or the small air filling factor. At large period $\Lambda$ value, it is recommended to make shallow nano-structure to improve light transmission. For the grating height (d) larger than $60 \mathrm{~nm}$, cylindrical nano-hole grating has the best performance.

In general, nano-hole structure can improve light extraction in a wider range of the grating height (d). Nano-hole is also easy to be achieved in our fabrication [11]. We furthermore study the nano-hole grating in detail $(\Lambda=140 \mathrm{~nm}, 180 \mathrm{~nm}, 220 \mathrm{~nm}$, and $260 \mathrm{~nm}$ as well), and shown in Fig. 4 . We find that the $\Lambda=140 \mathrm{~nm}$ case has the highest light extraction output. Compared to the no-grating case, the light extraction improvement is about $10 \%$ for the $230 \mathrm{~nm}$-depth and 120 -width grating. However, the smaller pattern size raises fabrication challenges. Actually, the large grating period is not an idea design for the conical pillar grating and the nano-hole grating. 


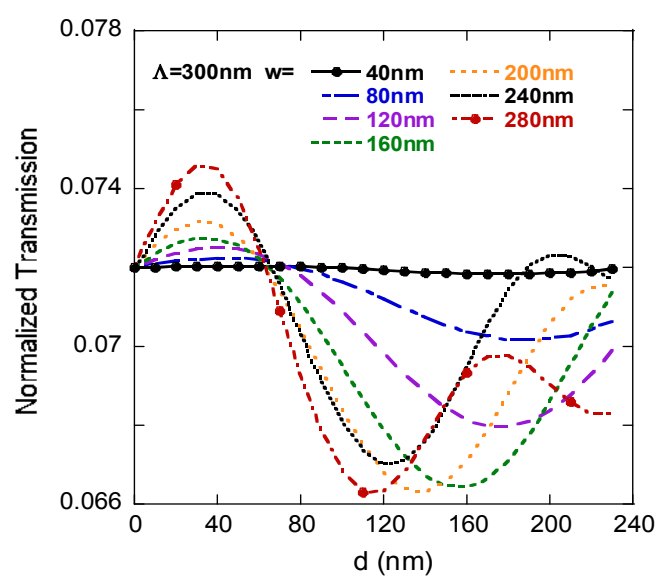

(a)

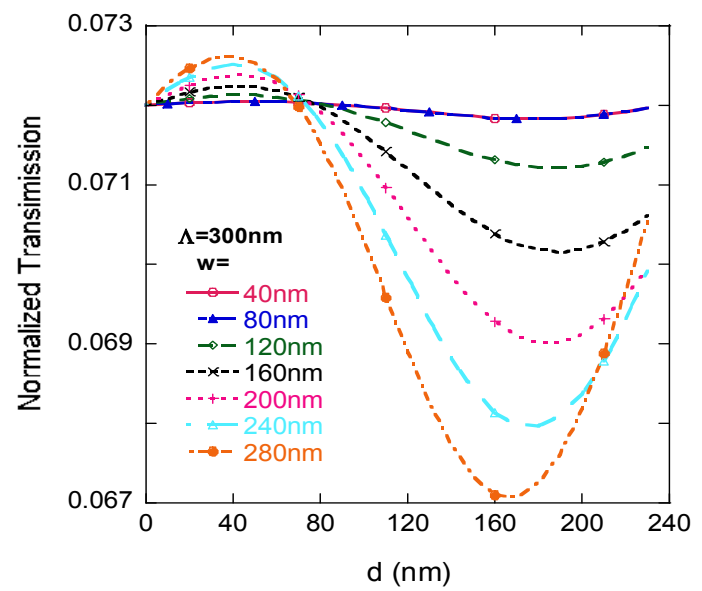

(b)

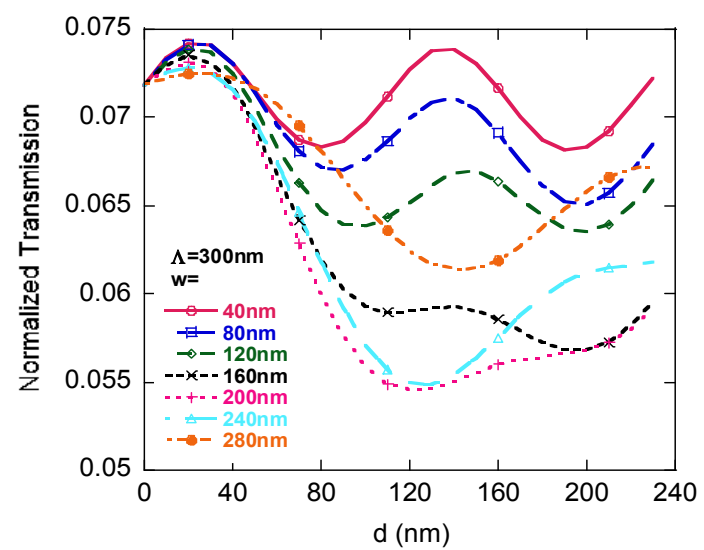

(c)

Figure 3. The simulation results a) cylindrical pillar grating, b) conical pillar grating, c) cylindrical nano-hole grating for grating period of $\Lambda=300 \mathrm{~nm}$.

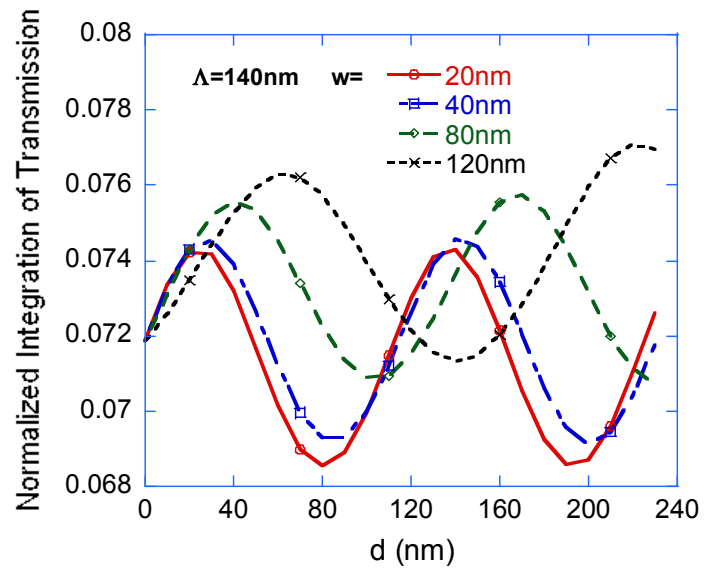

(a)

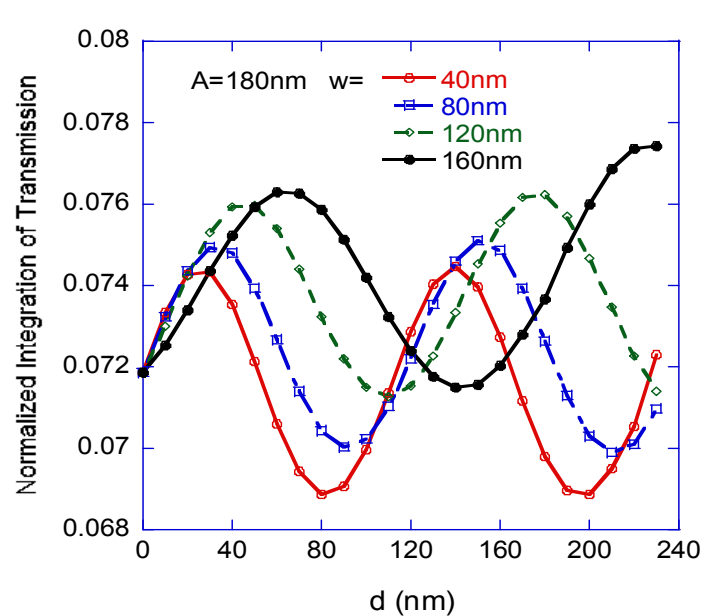

(b)

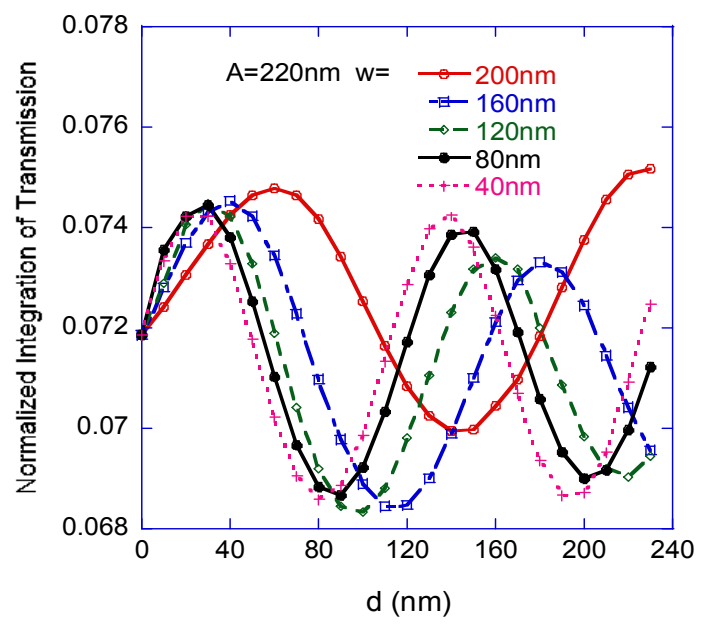

(c) 


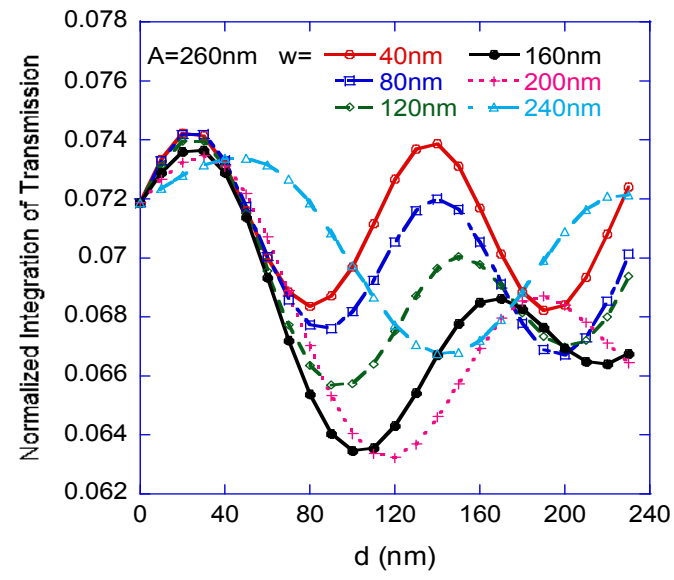

(d)

Fig. 4 Nano hole grating simulation results with $\Lambda=140,180,220,260 \mathrm{~nm}$.

\section{Conclusion}

In this paper, we present the simulation results of nano-scale grating design in GaN LEDs. We compare three different grating structures: cylindrical pillar grating, conical pillar grating, and cylindrical nanohole grating. And we show that the small grating period will yield more light extraction efficiency.

\section{Acknowledgement}

This work is sponsored by the Department of the Navy, Office of Naval Research, under Award \# N00014-07-1-1152 and "ChunHui" research fellow 2008, Ministry of Education, China. 973 ProgramNational Basic Research Program of China (2007CB307004); High Technology program (8632006AA03A113) and National Nature Science Foundation of China (60675032, 60577030 and 60607003).

\section{References}

[1] S. M. Huang , Y. Yao , C. Jin , Z. Sun , and Z. J. Dong, "Enhancement of the light output of GaN-based lightemitting diodes using surface-textured indium-tin-oxide transparent ohmic contacts", Displays, vol. 29, 2008, pp. 254-25,.

[2] G S.-H. Huang, R.-H. Horng, K.-S. Wen, Y.-F. Lin, K.W. Yen, and D.-S. Wuu, "Improved light extraction of Nitride-based flip-chip light-emitting diodes via Sapphire shaping and texturing", IEEE Photon. Tech. Lett., vol. 18, 2006, pp. 2623-2625.

[3] M.-K. Lee, C.-L. Ho, and P.-C. Chen, Light Extraction Efficiency Enhancement of GaN Blue LED by Liquid-PhaseDeposited ZnO Rods, IEEE Photon. Tech. Lett. vol 20, 2008, pp. 252-255.

[4] T. V. Cuong, H. S. Cheong, and C.-H. Hong, Calculation of the external quantum efficiency of light emitting diodes with different chip designs, Phys. Stat. Sol. (c) vol.1, 2004, pp. 2433-2440 .

[5] K. Bao, X.-N. Kang, B. Zhang, T. Dai, C. Xiong, H. Ji,G.-Y. Zhang, and Y. Chen, Improvement of Light Extraction from Micro-Pattern Encapsulated GaN-based LED by Imprinting IEEE Photon. Technol. Lett. vol. 19, 2007, pp.1840-1842.

[6] Rsoft Design Inc, http://www.rsoftdesign.com/.

[7] M. G. Moharam and T. K. Gaylord, "Rigorous coupledwave analysis of metallic surface-relief gratings", J. Opt. Soc. Am. A, vol. 3, 1986, pp. 1780-1788.

[8] L. Li, New formulation of the Fourier modal method for crossed surface-relief gratings, J. Opt. Soc. Am. A vol. 14, 1997, p. 2758.

[9] S. H. Kim, K.-D. Lee, J.-Y. Kim, M.-K. Kwon, and S.-J. Park, Fabrication of photonic crystal structures on light emitting diodes by nanoimprint lithography, TOP Nanotechnology, vol. 18, 2007, p. 055306.

[10] H.-G. Hong, S.-S. Kim, D.-Y. Kim, T. Lee, J.-O. Song, J. H. Cho, C. Sone, Y. Park, and T.-Y. Seonga, Enhancement of the light output of GaN-based ultraviolet light-emitting diodes by a one-dimensional nanopatterning process, App. Phy. Lett. 88, 2006, pp. 103505-103507.

[11] T. Dai, X.N. Kang, B. Zhang, Z.S. Zhang, D. Liu, X. Wang, K. Bao, X.N. Kang, J. Xu, D.P. Yu, and X. Zhu, "Surface Light Extraction Mapping from two-dimensional Array of 12-Fold Photonic Quasicrystal on Current Injection GaN-Based LEDs," Chin. Phys. Lett., vol. 24, no. 4, 2007, pp. 979-982. 